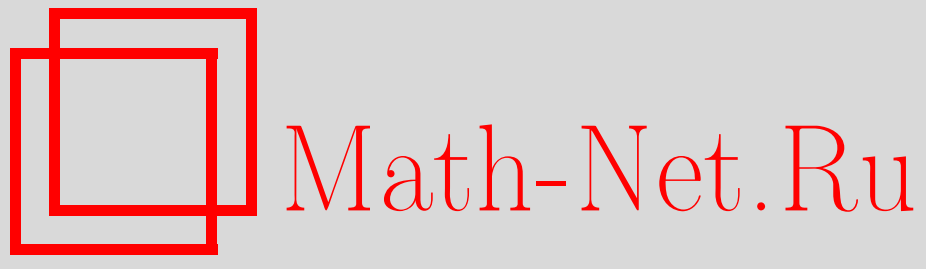

С. М. Маламуд, Аналог теоремы Пуанкаре о перемежаемости для нормальных матриц и теорема ГауссаЛукаса, Функи. анализ и его прил., 2003, том 37, выпуск $3,85-88$

DOI: https://doi.org/10.4213/faa162

Использование Общероссийского математического портала MathNet.Ru подразумевает, что вы прочитали и согласны с пользовательским соглашением

http://www . mathnet.ru/rus/agreement

Параметры загрузки:

IP: 3.91 .87 .62

26 апреля 2023 г., $17: 18: 27$

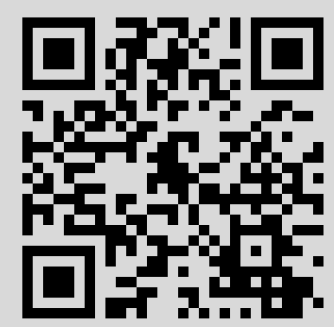


УДК $517+512.64$

\title{
Аналог теоремы Пуанкаре о перемежаемости для нормальных матриц и теорема Гаусса-Лукаса
}

\author{
(c) 2003. С. М. МАЛАМУД
}

1. Введение. В заметке найдены необходимые и достаточные условия для того, чтобы две последовательности чисел $\left\{\lambda_{j}\right\}_{1}^{m}$ и $\left\{\mu_{j}\right\}_{1}^{m-1}$ были спектрами нормальной $m \times m$-матрицы $A$ и ее главной подматрицы $A_{m-1}$, т. е. решены прямая и обратная спектральные задачи для пары $\left\{A, A_{m-1}\right\}$, где $A$ нормальна. В частности, получен аналог теоремы Пуанкаре о перемежаемости [10]. С помощью этого результата получено значительное усиление известной теоремы Гаусса-Лукаса о нулях производной многочлена [8], а также доказаны гипотезы де БрёйнаШпрингера [1] и Шёнберга [13].

2. Обобщение понятия мажоризации. Обозначим через $\operatorname{conv}(Y)$ выпуклую оболочку множества $Y\left(\subset \mathbb{R}^{n}\right)$, и пусть $M_{n}(\mathbb{K})-$ множество $n \times n$-матриц с элементами из поля $\mathbb{K}$, а $\sigma(A)-$ спектр матрицы $A \in M_{n}(\mathbb{C})$.

Пусть $x=\left\{x_{k}\right\}_{1}^{l}$ и $y=\left\{y_{k}\right\}_{1}^{m}$ - две последовательности векторов из $\mathbb{R}^{n}$, $x_{k}, y_{k} \in \mathbb{R}^{n}$ и $l \leqslant m$.

ОПРЕДЕЛЕНИЕ 1. Будем говорить, что последовательность $y=\left\{y_{k}\right\}_{1}^{m}$ мажорирует последовательность $\left\{x_{k}\right\}_{1}^{l}$ и писать $x \prec y$, если для каждого $p \in\{1, \ldots, l\}$ выполнены условия

$$
\operatorname{conv}\left(\sum_{k=1}^{p} x_{i_{k}}: 1 \leqslant i_{1}<\cdots<i_{p} \leqslant l\right) \subset \operatorname{conv}\left(\sum_{k=1}^{p} y_{i_{k}}: 1 \leqslant i_{1}<\cdots<i_{p} \leqslant m\right)
$$

Заметим, что при $l=m$ последнее из условий (1) принимает вид $x_{1}+\cdots+x_{m}=$ $y_{1}+\cdots+y_{m}$.

Напомним, что матрицу $S=\left(s_{i j}\right) \in M_{m}(\mathbb{R})$ называют бистохастической и пишут (см. [6]) $S \in \Omega_{m}$, если $s_{i j} \geqslant 0, i, j \in\{1, \ldots, m\}$, и сумма элементов каждой строки и каждого столбца равна единице.

Будем относить матрицу $S$ к классу $\Omega_{m}^{u}\left(\subset \Omega_{m}\right)$ унитарно стохастических матриц, если $S=\left(\left|u_{i j}\right|^{2}\right)_{i, j=1}^{m}$, где $U=\left(u_{i j}\right)_{i, j=1}^{m}$ унитарна.

ОПРЕДЕЛЕНИЕ 2. Будем говорить, что последовательность $y=\left\{y_{k}\right\}_{1}^{m}$ бистохастически мажорирует последовательность $x=\left\{x_{k}\right\}_{1}^{l}$ и писать $x \prec_{b s} y$, если существуют векторы $x_{l+1}, \ldots, x_{m} \in \mathbb{R}^{n}$ и бистохастическая матрица $S \in \Omega_{m}$, такие, что $\tilde{x}:=\left\{x_{k}\right\}_{1}^{m}=\left(S \otimes I_{n}\right) y$. Если в этом определении $S \in \Omega_{m}^{u}$, то будем писать $x \prec u b s y$.

ПрЕДЛОЖЕНИЕ 3. Пусть $x_{j}, y_{j} \in \mathbb{R}^{n}$ при $j \in\{1, \ldots, m\}$ u $x=\left(x_{1}, \ldots, x_{m}\right)$, $y=\left(y_{1}, \ldots, y_{m}\right) \in \mathbb{R}^{n m}$. Для каждой бистохастической матрицьь $S \in \Omega_{m}$ верно соотношение $\left(S \otimes I_{n}\right) y \prec y$, т. е. из условия $x \prec_{b s} y$ вытекает условие $x \prec y$.

При $n=1$, т. е. для последовательностей вещественных чисел, определение 1 эквивалентно классическому определению из $[6,9]$. Эквивалентность определений 1 и 2 (т.е. условий $x \prec_{b s} y$ и $x \prec y$ ) при $n=1$ вытекает из теоремы Харди-Литтлвуда-Пойи (см. [5-9]). 
Следующий пример показывает, что при $n>1$ определения 1 и 2 не эквивалентны, и дает негативный ответ на вопрос из [7, p. 434].

ПримеР 4. Пусть

$$
\begin{aligned}
& x=\left(x_{1}, x_{2}, x_{3}, x_{4}\right)=((12,12),(12,12),(5,3),(3,5)), \\
& y=\left(y_{1}, y_{2}, y_{3}, y_{4}\right)=((8,16),(16,8),(0,0),(8,8)) .
\end{aligned}
$$

Элементарно показывается, что $x \prec y$. Так как, однако, $x_{1}$ принадлежит стороне $\left[y_{1}, y_{2}\right]$ треугольника $y_{1} y_{2} y_{3}=\operatorname{conv}(y)$, то его представление $x_{1}=\left(y_{1}+y_{2}\right) / 2$ в виде выпуклой комбинации точек из $\left\{y_{k}\right\}_{1}^{4}$ единственно. Поэтому если существует бистохастическая матрица $S$, такая, что $x=S y$, то она имеет вид

$$
S=\left(\begin{array}{cccc}
1 / 2 & 1 / 2 & 0 & 0 \\
1 / 2 & 1 / 2 & 0 & 0 \\
0 & 0 & s_{33} & s_{34} \\
0 & 0 & s_{43} & s_{44}
\end{array}\right) .
$$

Это, однако, невозможно, так как $x_{3}, x_{4} \notin \operatorname{conv}\left\{y_{3}, y_{4}\right\}$.

3. Аналог теоремы Пуанкаре о перемежаемости. Пусть $A=A^{*} \in M_{m}(\mathbb{C})$, $\sigma(A)=\left\{\lambda_{1} \leqslant \ldots \leqslant \lambda_{m}\right\}$ и $A_{m-1}-$ подматрица матрицы $A$, полученная из нее удалением последних строки и столбца. Пусть, далее, $\sigma\left(A_{m-1}\right)=\left\{\mu_{1} \leqslant \ldots \leqslant\right.$ $\left.\mu_{m-1}\right\}$. Известная теорема Пуанкаре о перемежаемости [10] (она же - теорема Коши [6]) утверждает, что наборы $\left\{\lambda_{k}\right\}_{1}^{m}$ и $\left\{\mu_{k}\right\}_{1}^{m-1}$ перемежаются, т. е.

$$
\lambda_{1} \leqslant \mu_{1} \leqslant \lambda_{2} \leqslant \ldots \leqslant \mu_{m-1} \leqslant \lambda_{m} .
$$

Справедливо также (см. $[10,2])$ обратное утверждение: для двух перемежающихся наборов чисел $\left\{\lambda_{k}\right\}_{1}^{m}$ и $\left\{\mu_{k}\right\}_{1}^{m-1}$ существует матрица $A=A^{*} \in M_{m}(\mathbb{C})$, такая, что $\sigma(A)=\left\{\lambda_{k}\right\}_{1}^{m}$ и $\sigma\left(A_{m-1}\right)=\left\{\mu_{k}\right\}_{1}^{m-1}$.

Оказывается, что для нормальной матрицы $A$ спектр ее подматрицы $A_{m-1}$ также можно охарактеризовать чисто геометрически.

Ниже, используя определения мажоризации для комплексных чисел, мы отождествляем $\mathbb{C}$ с $\mathbb{R}^{2}$. Далее, по каждому вектору $\left\{\lambda_{j}\right\}_{1}^{k} \in \mathbb{C}^{k}$ определим семейство

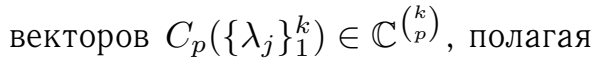

$$
C_{p}\left(\left\{\lambda_{j}\right\}_{1}^{k}\right):=\left\{\lambda_{i_{1}} \lambda_{i_{2}} \cdots \lambda_{i_{p}}\right\}_{1 \leqslant i_{1}<i_{2}<\cdots<i_{p} \leqslant k}, \quad p \in\{1, \ldots, k\} .
$$

Теорема 5. Пусть $\left\{\lambda_{j}\right\}_{1}^{m} \in \mathbb{C}^{m},\left\{\mu_{j}\right\}_{1}^{m-1} \in \mathbb{C}^{m-1}$. Тогда для существования нормальной матрищь $A$, такой, что $\sigma(A)=\left\{\lambda_{j}\right\}_{1}^{m}$ u $\sigma\left(A_{m-1}\right)=\left\{\mu_{j}\right\}_{1}^{m-1}$, необходимо, чтобы условия

$$
C_{p}\left(\left\{\mu_{j}-\alpha\right\}_{1}^{m-1}\right) \prec_{u b s} C_{p}\left(\left\{\lambda_{j}-\alpha\right\}_{1}^{m}\right)
$$

выполнялись при $\alpha \in \mathbb{C}, p \in\{1, \ldots, m-1\}$, и достаточно их выполнения при $p=m-1 u \alpha \in\left\{\lambda_{1}, \ldots, \lambda_{m}\right\}$.

СледствиЕ 6. Для существования нормальной матриць $A$, такой, ито $\sigma(A)=\left\{\lambda_{j}\right\}_{1}^{m}$ и $\sigma\left(A_{m-1}\right)=\left\{\mu_{j}\right\}_{1}^{m-1}$, необходимо и достаточно, чтобы

$$
q_{1}\left(\lambda_{k}\right) \in \operatorname{conv}\left\{0, q^{\prime}\left(\lambda_{k}\right)\right\} \quad \text { npu } k \in\{1, \ldots, m\},
$$

где $q(\lambda):=\prod_{j=1}^{m}\left(\lambda-\lambda_{j}\right)$ u $q_{1}(\lambda):=\prod_{j=1}^{m-1}\left(\lambda-\mu_{j}\right)$. 
ЗАмЕЧАНИЕ 7. Полагая $\alpha=\lambda_{k}$ в последнем из условий $(3)$ при $p=m-1$, получим, что числа $q_{1}\left(\lambda_{k}\right)\left(q^{\prime}\left(\lambda_{k}\right)\right)^{-1}$ неотрицательны. Этот факт в случае вещественных наборов чисел $\left\{\lambda_{j}\right\}_{1}^{m}$ и $\left\{\mu_{j}\right\}_{1}^{m-1}$ эквивалентен их перемежаемости.

ЗАмЕчАНИЕ 8 . При $m=3$ отношения мажоризации $\prec$ и $\prec_{b s}$ эквивалентны (но не эквивалентны отношению $\left.\prec_{u b s}\right)$. Заметим, что при $m=3$ более слабая, чем в (3), мажоризация $\prec_{b s}$ означает в точности, что

$$
\begin{aligned}
& \mu_{1}, \mu_{2} \in \operatorname{conv}\left\{\lambda_{j}\right\}_{1}^{3}, \quad \mu_{1}+\mu_{2} \in \operatorname{conv}\left\{\lambda_{1}+\lambda_{2}, \lambda_{1}+\lambda_{3}, \lambda_{2}+\lambda_{3}\right\}, \\
& \mu_{1} \mu_{2}-\alpha\left(\mu_{1}+\mu_{2}\right) \in \operatorname{conv}\left\{\lambda_{k} \lambda_{p}-\alpha\left(\lambda_{k}+\lambda_{p}\right)\right\}_{1 \leqslant k<p \leqslant 3}, \quad \alpha \in \mathbb{C} .
\end{aligned}
$$

В доказательстве теоремы 5 используются некоторые идеи и методы из [2-4], а также аппарат внешней алгебры.

4. О теореме Гаусса-Лукаса и гипотезе де Брёйна-Шпрингера. Согласно классической теореме Гаусса-Лукаса (см. [8]), корни производной многочлена лежат в выпуклой оболочке его корней. Различным усилениям этой теоремы посвящен ряд работ (см., например, $[11,12]$ и цитируемую там литературу).

Пусть, далее, $q(\in \mathbb{C}[z])$ - полином степени $m$ с корнями $\left\{\lambda_{j}\right\}_{1}^{m}$, а $\left\{\mu_{j}\right\}_{1}^{m-1}-$ корни его производной. Полагая $\mu_{m}:=\left(\sum_{1}^{m} \lambda_{j}\right) / m$, можно переформулировать теорему Гаусса-Лукаса так: существует стохастическая (по строкам) матрица $S^{\prime}$, такая, что $\left\{\mu_{j}\right\}_{1}^{m}=S^{\prime}\left\{\lambda_{j}\right\}_{1}^{m}$.

Из теоремы 5 с учетом очевидного тождества $q^{\prime}(z) / q(z)=\sum 1 /\left(z-\lambda_{j}\right)$ и замечания 1 вытекает более полный результат.

ПрЕДЛОЖЕНИЕ 9. Наборы $\left\{\lambda_{j}\right\}_{1}^{m} u\left\{\mu_{j}\right\}_{1}^{m-1}$ удовлетворяют условиям (3) для всех $\alpha \in \mathbb{C} u p \in\{1, \ldots, m-1\}$.

Однако в этом случае можно получить значительно более полную информацию о матрицах $S \in \Omega_{m}^{u}$, реализующих мажоризацию.

Теорема 10. Пусть $p \in\{1, \ldots, m-1\}, a:=\left(\begin{array}{c}m-1 \\ p\end{array}\right), b:=\left(\begin{array}{c}m \\ p\end{array}\right)$. Tогда cyщ̧eствует матрица $S_{p}=\left(s_{i j p}\right) \in \Omega_{b}^{u}$, такая, что $\sum_{i=a+1}^{b} s_{i j p}=p / m$ для всех $j \leqslant b$ u $C_{p}\left(\left\{\mu_{j}-\alpha\right\}_{1}^{m-1}\right)=P S_{p} C_{p}\left(\left\{\lambda_{j}-\alpha\right\}_{1}^{m}\right) \quad(c м .(2))$, где $P: \mathbb{C}^{b} \rightarrow \mathbb{C}^{a}-$ естественный ортопроектор.

В частности, при $p=1$ найдется $S_{1} \in \Omega_{m}^{u}$, такая, ито $s_{m j 1}=1 / m$, $j \in\{1, \ldots, m\}, u\left\{\mu_{j}\right\}_{1}^{m}=S_{1}\left\{\lambda_{j}\right\}_{1}^{m}$.

Де Брёйн и Шпрингер высказали в [1] следующую гипотезу: для любой выпуклой функции $f: \mathbb{C} \rightarrow \mathbb{R}$ справедливо неравенство

$$
\frac{\sum f\left(\mu_{j}\right)}{m-1} \leqslant \frac{\sum f\left(\lambda_{j}\right)}{m}
$$

Им удалось доказать это неравенство лишь для некоторого класса выпуклых функций. Теорема 10 позволяет доказать более сильное утверждение, совпадающее с неравенством (4) при $p=1$.

ТЕОРЕмА 11. Для каждого $p \in\{1, \ldots, m-1\}$ и любой выпуклой функции $f: \mathbb{C} \rightarrow \mathbb{R}$ верно неравенство

$$
\frac{1}{\left(\begin{array}{c}
m-1 \\
p
\end{array}\right)} \sum_{1 \leqslant i_{i}<\cdots<i_{p} \leqslant m-1} f\left(\prod_{j=1}^{p}\left(\mu_{i_{j}}-\alpha\right)\right) \leqslant \frac{1}{\left(\begin{array}{c}
m \\
p
\end{array}\right)} \sum_{1 \leqslant i_{1}<\cdots<i_{p} \leqslant m} f\left(\prod_{j=1}^{p}\left(\lambda_{i_{j}}-\alpha\right)\right) .
$$


5. Гипотеза Шёнберга. Следующая теорема сформулирована Шёнбергом [13] в качестве гипотезы.

Теорема 12. Пусть $\left\{\lambda_{j}\right\}_{1}^{m}-$ корни полинома $q(\in \mathbb{C}[z])$ степени $m, a\left\{\mu_{j}\right\}_{1}^{m-1}$ - корни его производной. Если $\sum_{j=1}^{m} \lambda_{j}=0$, то

$$
m \sum_{j=1}^{m-1}\left|\mu_{j}\right|^{2} \leqslant(m-2) \sum_{j=1}^{m}\left|\lambda_{j}\right|^{2},
$$

причем равенство имеет место в точности тогда, когда числа $\left\{\lambda_{j}\right\}_{1}^{m}$ лежат на одной прямой.

Поясним идею доказательства. Пусть $A:=U \Lambda U^{*}$, где $\Lambda:=\operatorname{diag}\left(\lambda_{1}, \ldots, \lambda_{m}\right)$, $U=m^{-1 / 2}\left(\varepsilon^{k(j-1)}\right)_{k, j=1}^{m}$ и $\varepsilon=e^{2 \pi i / m}$. Можно показать, что матрица $A$ дает решение обратной задачи для наборов $\left\{\lambda_{j}\right\}_{1}^{m}$ и $\left\{\mu_{k}\right\}_{1}^{m-1}$, т. е. $\sigma(A)=\left\{\lambda_{j}\right\}_{1}^{m}$ и $\sigma\left(A_{m-1}\right)=\left\{\mu_{k}\right\}_{1}^{m-1}$. Легко видеть, что $A=m^{-1}\left(r\left(\varepsilon^{k-j}\right)\right)_{k, j=1}^{m}$, где $r(z):=$ $\sum_{j=1}^{m} \lambda_{j} z^{j-1}$. Отсюда выводится тождество $m\left\|A_{m-1}\right\|_{2}^{2}=(m-2)\|A\|_{2}^{2}$, в котором $\|A\|_{2}:=m^{-1}\left(\sum_{k, j}\left|r\left(\varepsilon^{k-j}\right)\right|^{2}\right)^{1 / 2}-$ фробениусова норма матрицы. Осталось заметить, что $m \sum_{j=1}^{m-1}\left|\mu_{j}\right|^{2} \leqslant m\left\|A_{m-1}\right\|_{2}^{2}=(m-2)\|A\|_{2}^{2}=(m-2) \sum_{j=1}^{m}\left|\lambda_{j}\right|^{2}$.

Благодарность. Я глубоко признателен $\Phi$. В. Петрову за ценные замечания, позволившие значительно улучшить текст статьи, а также за информацию о работах [1] и [12].

\title{
ЛитеРАТУРА
}

1. de Bruijn N. G., Springer T. A. Indag. Math., 9, 264-270 (1947). 2. Маламуд M. М. Укр. матем. ж., 44, №12, 1658-1688 (1992). 3. Malamud S. M. Linear Algebra Appl., 322, 19-41 (2001). 4. Маламуд С. М. Матем. заметки, 69, № 4, 633-637 (2001). 5. Маркус А. С. УМН, 19, вып. 4 (118), 93-123 (1965). 6. Маркус М., Минк Х. Обзор по теории матриц и матричных неравенств. Наука, M., 1972. 7. Marshall A. W., Olkin I. Inequalities: Theory of Majorization and Its applications. Academic Press, 1979. 8. Полиа Г., Сеге Г. Задачи и теоремы из анализа. Наука, М., 1978. 9. Харди Г., Литтлвуд Дж., Пойа Г. Неравенства. ИЛ, М., 1948. 10. Horn R., Johnson Ch. Matrix Analysis, Cambridge., 1986. 11. Pawlowski P. Trans. Amer. Math. Soc., 350, No. 11, 4461-4472 (1998). 12. Schmeisser G. In: Approximation Theory: A volume dedicated to Blagovest Sendov, DARBA, Sofia, 2002, pp. 353-369. 13. Schoenberg I. Amer. Math. Monthly, 93, 8-13 (1986).

Eidgenössische Technische Hochschule (ETH), Zürich e-mail: semka@math.ethz.ch

Поступило в редакцию 1 октября 2002 г.

УДК 514.75

\section{Об устойчивости бифуркационных диаграмм исчезающих уплощений}

\author{
(C) 2003. Р. УРИБЕ-ВАРГАС
}

1. Введение. Недавно В. И. Арнольд, исследуя особенности малых возмущений сильно вырожденных лагранжевых и лежандровых отображений (исчезающие особенности) получил различные модификации классической теоремы о че- 